\title{
RELAXATION FREQUENCY SHIFT IN TRIGLYCINE SULFATE UNDER THE INFLUENCE OF SILICA NANOPARTICLES AT LOW FREQUENCIES
}

\author{
MAI BICH DUNG ${ }^{1}$, NGUYEN HOAI THUONG ${ }^{2}$ \\ ${ }^{1}$ Institute of Biotechnology and Food Technology, Industrial University of Ho Chi Minh City \\ ${ }^{2}$ Faculty of Electrical Engineering Technology, Industrial University of Ho Chi Minh City \\ nguyenthuongfee@iuh.edu.vn
}

\begin{abstract}
The present work is devoted to clarifying the influence of silica nanoparticles on dielectric relaxation frequencies of a classical ferroelectric - triglycine sulfate at low frequencies $\left(10^{2}-10^{7} \mathrm{~Hz}\right)$ from $20{ }^{\circ} \mathrm{C}$ to phase transition point for composite samples prepared at different composition weight ratios. The results indicated the reduction of relaxation frequency with increasing the silica content due to the intensified interaction between nanoparticles and tryglycine sulfate inclusion. The nature of this interaction was thoroughly discussed in this study.
\end{abstract}

Keywords. Ferroelectric nanocomposites, dielectric relaxation, triglycine sulfate, silica nanoparticles, hydrogen bonds.

\section{INTRODUCTION}

Technological development cannot be separated from discovering new promising materials to meet strict requirements from manufacturers. Along with intelligent properties, the size of materials must be paid attention in relation to the integration ability into modern electronics devices. In this regard, nanomaterials can fill the gap.

Ferroelectrics play an important role in modern electronics due to their tunable electrophysical properties that can be controlled by external electric fields or mechanical stresses. The most valuable region is ferroelectric phase, in which the domain walls always exist and can be used for manufacturing memories and related applications. Nowadays, along with modern fabrication techniques, ferroelectrics-related materials are found in the form of thin films or nanocomposites with advanced characteristics created by size effects at nanoscale level and the interaction between composite components. Up to now, state-of-theart ferroelectric nanocomposites have been made. However, the overall picture still has not been established and the published works are not commensurate with the development potential of this kind of material.

Triglycine sulfate (TGS) is one of the most important classical ferroelectrics used in various applications as pyroelectric detection, thermal imaging devices, sensitive detectors, transducers, memories, etc. [1,2]. However, as other primitive ferroelectrics, TGS single crystals have several drawbacks with low temperature of phase transition $\left(49^{\circ} \mathrm{C}\right)$. Besides, the high mobility of domain walls at room temperature could be interesting challenges to overcome for expanding its application scope. Despite the fact that properties of primitive ferroelectrics are mostly completely explored and no longer attracts researchers, their combination with dielectrics can lead to the formation of promising anomalies for electronics. For examples, the introduction of TGS into nanopores of cellulose [3-8], $\mathrm{Al}_{2} \mathrm{O}_{3}$ [9] and silicon oxide [9] resulted in the expansion of ferroelectric phase, the diffusion of phase transition or low motion of domain walls.

Along with silicon - a heart of modern electronics, silica or silicon dioxide $\left(\mathrm{SiO}_{2}\right)$ is known as an ideal dielectric used for fabricating electronics materials in the role of a reinforcing component. Besides, $\mathrm{SiO}_{2}$ in the form of nanoparticles can be also utilized for adjusting ferroelectric properties through the interaction of $\mathrm{SiO}_{2}$ with fillers due to large specific area and hydrophilicity [10]. Besides, another interesting aspect of $\mathrm{SiO}_{2}$ is related to the ability to contain residual water even at considerable high temperature (up to $500{ }^{\circ} \mathrm{C}$ ). It means that the hydrogen bonds could occur and interact with other hydrogen-containing ferroelectrics [10]. Meanwhile, triglycine sulfate is a hydrogen-bonded ferroelectric and its ferroelectricity is conditioned 
by characteristics of hydrogen systems. In other words, the intervention of hydrogen bonds in TGS can lead to the appearance of new properties that could be useful for practical applications.

The composite from triglycine sulfate and $\mathrm{SiO}_{2}$ nanoparticles has been synthesized already as shown in $[11,12]$. However, there are some aspects that have not clarified: (i) the works $[11,12]$ presented the experimental results for investigation of phase transition only, there were no comments on the relaxation anomalies regardless of that the frequency effects are important for manufacturing electronics devices; (ii) the influence of composition weight ratio on relaxation behavior was not considered at all. In this regard, the present study is to fill this gap.

In this study, the composite form TGS mixed with $\mathrm{SiO}_{2}$ nanoparticles was prepared at different composition weight ratios for testing frequency dependences of dielectric characteristics at different temperatures from $20^{\circ} \mathrm{C}$ to phase transition point in low frequency range under a weak electric field (1 $\mathrm{V} / \mathrm{cm}$ ). The obtained results are thoroughly discussed based on the assumption of strong influence of $\mathrm{SiO}_{2}$ on the domain-wall motion in TGS component.

\section{MATERIAL PREPARATION AND EXPERIMENTAL METHODS}

The starting $\mathrm{SiO}_{2}$ nanoparticles in size of $20-200 \mathrm{~nm}$ prepared by sol-gel method was stored in the form of nanodispersered hydrosol before utilizing for preparation of the composite. The size distribution was evaluated using a Zetasizer analyzer as reported in [11]. The TGS used for preparation was supplied by Merck company with the purity higher $99 \%$ confirmed by XRD and FTIR data for that the strange phase and functional groups were not detected [11].

Composite samples at different $\mathrm{SiO}_{2}$ /TGS mass ratios were fabricated according the procedure described in detail in [11]. In the present study, we chose three mass ratios of $\mathrm{SiO}_{2}$ :TGS $=0.2: 1,1: 1$ and $3: 1$ to synthesize. Briefly, the composite was prepared by the mixing technique with a determined amount of starting materials of $\mathrm{SiO}_{2}$ and TGS while stirring was kept in closed bar to avoid water evaporation and then in open air for partially removing residual water from initial mixture. The growth of TGS crystals in the composite took place in 3 days before heating at $120{ }^{\circ} \mathrm{C}$ in $2 \mathrm{~h}$, crushing into fine powder and compressing into tablets. After putting silver electrode on large surface of tablets, the samples were ready for dielectric measurements.

The dielectric response of the synthesized composite under frequency was tested on Impedance/GainPhase Analyzer (SOLARTRON 1260A) in a weak electric field with an amplitude of $1 \mathrm{~V} \cdot \mathrm{cm}^{-1}$ from $10^{3}$ to $10^{6} \mathrm{~Hz}$. This experimental system allows us to smoothly adjust frequency step automatically. Besides, the modern thermostat with sample holder connected to the above system was able to heat up samples with the temperature error of $0.01 \mathrm{~K}$. The heating rate was established at $0.5^{\circ} \mathrm{C} \cdot \mathrm{min}^{-1}$. Because the relaxation of domain walls was quite long, the temperature was kept unchanged 30 minutes after conducting experiment for each frequency. The measurement error did not exceed $1 \%$. All data were automatically recorded on the computer.

\section{EXPERIMENTAL RESULTS AND DISCUSSION}

All characterization results were reported in detail in a previous study [11] in which the features of crystalline structures and functional groups have been explored to confirm the reliability of the synthesized composites using XRD and FTIR techniques.

The present work shows only the experimental data for the frequency dependences of dielectric parameters as the real and imaginary parts along with Cole-Cole diagrams used for analyzing the obtained data. The temperature range was chosen in ferroelectric phase because the dielectric relaxation in ferroelectric phase attract great attention from researcher thanks to their promising applications in practice. In paraelectric phase, the results didn't show any anomalies and therefore will not be presented in this study.

According the reported results [11], the ferroelectric phase of $\mathrm{SiO}_{2}$-TGS composite was significantly expanded with increasing the $\mathrm{SiO}_{2}$ content. Besides, the phase transition peaks became more diffused. In addition, the phase transition temperatures were 64,80 and $104{ }^{\circ} \mathrm{C}$ corresponding to the mass ratios of $\mathrm{SiO}_{2}: \mathrm{TGS}=0.2: 1,1: 1$ and 3:1, respectively. 

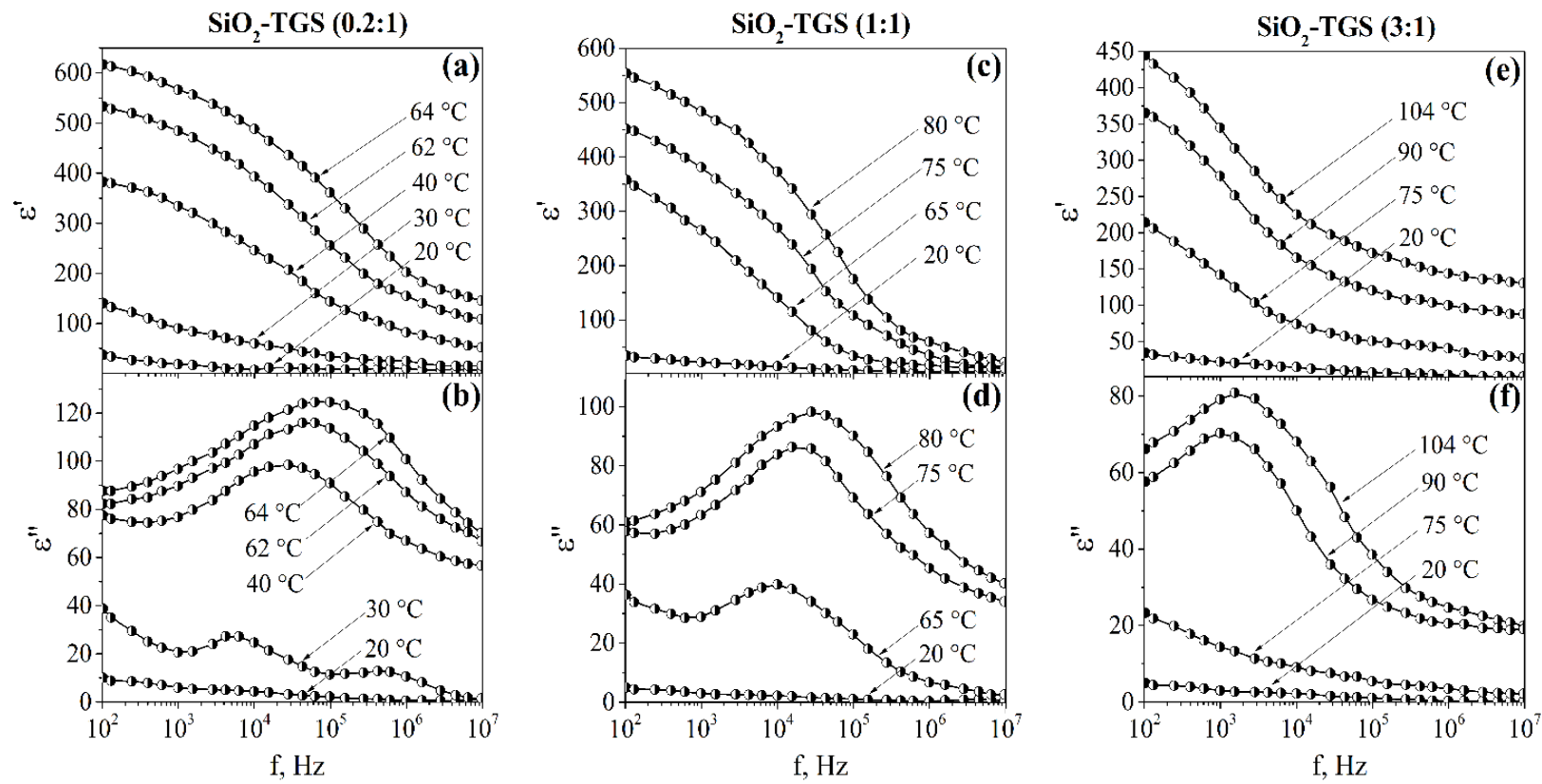

Figure 1: Frequency dependences of the real and imaginary parts of complex dielectric constant for $\mathrm{SiO}_{2}+\mathrm{TGS}$ composite at $\mathrm{SiO}_{2}$ :TGS weight ratios of 0.2:1 (a, b), 1:1 (c, d) and 3:1 (e, f) at different temperatures in ferroelectric phase.

The frequency dependences of the real $\varepsilon^{\prime}(f)$ and imaginary $\varepsilon^{\prime \prime}(f)$ parts of the complex dielectric permittivity as

$$
\varepsilon^{*}(f)=\varepsilon^{\prime}(f)+\mathrm{i} \varepsilon^{\prime \prime}(f)
$$

are presented in Fig. 1 at different temperatures from room temperature to phase transition point for composite samples prepared with different composition weight ratios. To be convenient for analysis, the corresponding Cole-Cole diagrams of $\varepsilon^{\prime \prime}\left(\varepsilon^{\prime}\right)$ were also plotted (Fig. 2).

It can be seen in Fig. 1 that the shape of $\varepsilon^{\prime}(f)$ and $\varepsilon^{\prime \prime}(f)$ are similar to those of single crystals TGS at low frequencies with a monotonic reduction of $\varepsilon^{\prime}$ observed in the presence of relaxation peaks in $\varepsilon^{\prime \prime}(f)$ $[13,14]$. Besides, the similarity was also shown in Cole-Cole diagrams with linear and semicircle shape of $\varepsilon^{\prime \prime}\left(\varepsilon^{\prime}\right)$ dependences at lower and higher frequencies, respectively (Fig. 2). This behavior was observed in composite from nanocrystalline cellulose and TGS inclusion [7]. However, there were several anomalies which must be paid attention here. Firstly, the positions of relaxation peaks $\varepsilon^{\prime \prime}(f) \mathrm{SiO}_{2}-\mathrm{TGS}$ composite for all studied samples shifted toward lower frequencies as compared to that detected in single crystals TGS $\left(10^{5}-10^{7} \mathrm{~Hz}\right.$ [14]). Indeed, the relaxation frequencies as seen in Fig. 1 were lower $100 \mathrm{kHz}\left(\mathrm{SiO}_{2}: \mathrm{TGS}=\right.$ 0.2:1) (Fig. 1b), $2.9 \mathrm{kHz}\left(\mathrm{SiO}_{2}: \mathrm{TGS}=1: 1\right)$ (Fig. 1d) and $1.6 \mathrm{kHz}\left(\mathrm{SiO}_{2}: \mathrm{TGS}=3: 1\right)$ (Fig. 1d). Secondly, the composition weight ratios strongly affected the obtained relaxation frequencies of the $\mathrm{SiO}_{2}$-TGS. The higher the $\mathrm{SiO}_{2}$ content was, the lower the relaxation frequency took place. In Figure 2, the linear dispersion took advantages over Cole-Cole relaxation with increasing $\mathrm{SiO}_{2}$ content. For example, at room temperature for $\mathrm{SiO}_{2}$ :TGS = 3:1, there was only the linear dispersion observed without the presence of Cole-Cole arc (Fig. 2c). At higher temperatures, Cole-Cole relaxation became more pronounced while linear dispersion was less and totally disappeared in the vicinity of phase transition point. Thirdly, the linear dispersion of the composite was observed in higher-frequency region (Fig. 2) as compared to those of single crystals TGS $(<10 \mathrm{~Hz})[15]$. 
(a)
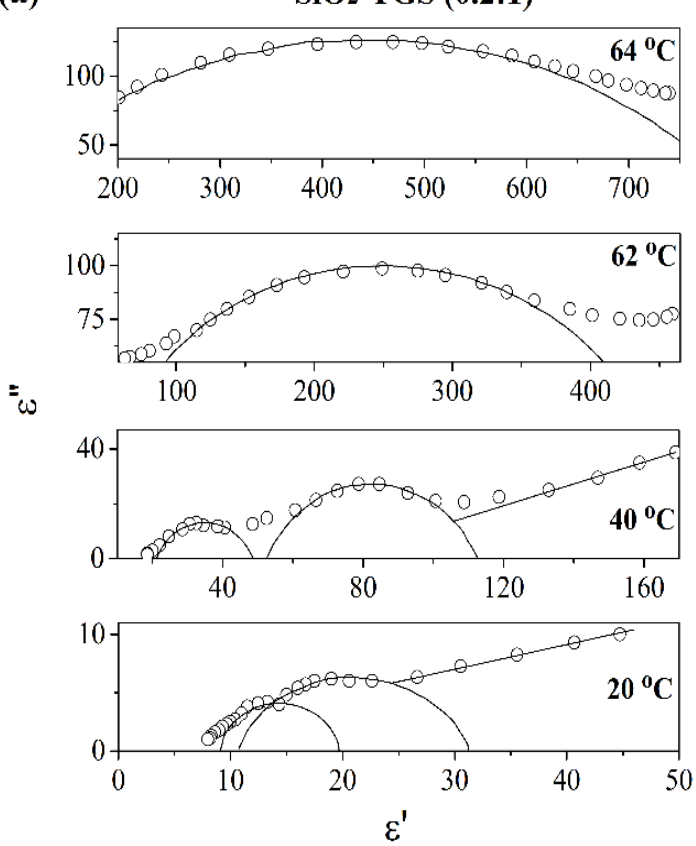

(b)
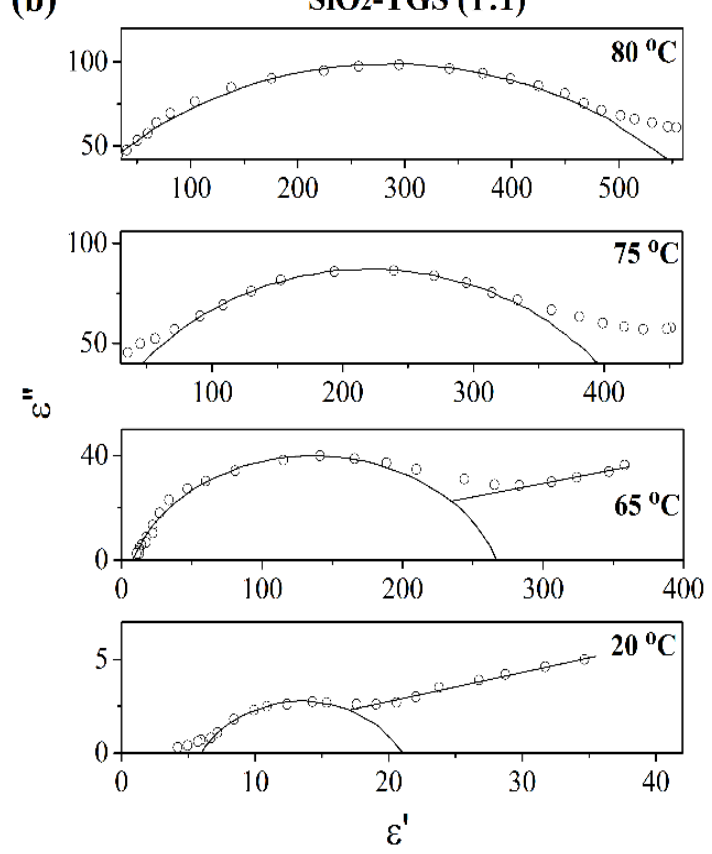

(c)

$\mathrm{SiO} 2-\mathrm{TGS}(3: 1)$
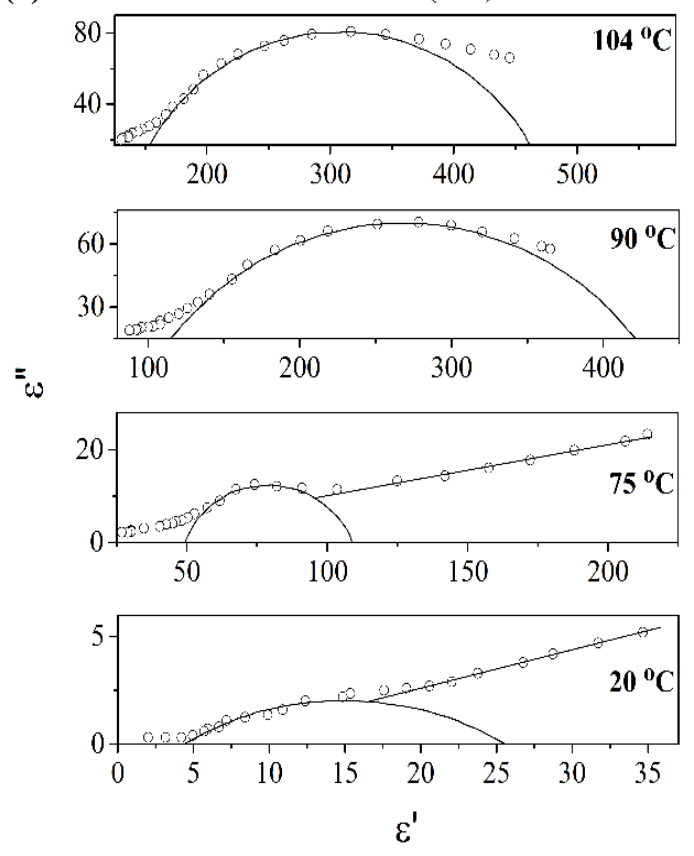

Figure 2: Cole-Cole diagrams for $\mathrm{SiO}_{2}+\mathrm{TGS}$ composite with $\mathrm{SiO}_{2}$ :TGS weight ratios of 0.2:1 (a), 1:1 (b) and 3:1 (c) at different temperatures in ferroelectric phase.

It should be noted that, for samples with low $\mathrm{SiO}_{2}$ content $\left(\mathrm{SiO}_{2}\right.$ :TGS $\left.=0.2: 1\right)$, an additional peak at $10^{5}-10^{6} \mathrm{~Hz}$ was observed at temperatures lower $40{ }^{\circ} \mathrm{C}$. With further increase in temperature, this peak gradually disappeared, probably, due to overlapping of the main relaxation peak at higher temperatures. The nature of this peak could be related to the presence of bulk TGS part in the composite. Indeed, in temperature dependences of dielectric constant, the volume part of TGS led to the appearance of an additional peaks at $49{ }^{\circ} \mathrm{C}$ i.e. coinciding with the Curie point of TGS single crystals [11]. It should be noted that the described anomalies were not detected in $\mathrm{SiO}_{2}$ without TGS. For pure $\mathrm{SiO}_{2}$, the values of dielectric constant and loss were quite small as compared to those of pure TGS or $\mathrm{SiO}_{2}-\mathrm{TGS}$ nanocomposites. 
The results presented in Fig. 1a, c, e also indicate that the values of dielectric constant and dielectric loss decreased with increasing $\mathrm{SiO}_{2}$ content. The relaxation peaks were more blurred in comparison with those of TGS single crystals [14]. The nature of these anomalies will be thoroughly discussed in the present work.

\section{DISCUSSION}

The influence of $\mathrm{SiO}_{2}$ on TGS could also result in the anomalies of dielectric relaxation. Known [15] that the higher-frequency Cole-Cole relaxation and lower-frequency linear dispersion of singles crystals TGS are relatively related to the reversible (equation 2) and irreversible (equation 3) domain-wall motion as follows:

$$
\begin{gathered}
\varepsilon^{*}-\varepsilon_{\infty \mathrm{Col}}=\frac{\Delta \varepsilon}{1+\left(j \omega \tau_{m}\right)^{1-\alpha}} \\
\varepsilon^{*}-\varepsilon_{\infty \text { lin }}=\varepsilon_{\infty \text { lin }}\left(j \omega \tau_{\text {lin }}\right)^{-\alpha_{\text {lin }}}
\end{gathered}
$$

where $\Delta \varepsilon=\varepsilon_{o C o l}-\varepsilon_{\infty} \mathrm{Col}$ - the depth of dispersion, $\varepsilon_{o C o l}, \varepsilon_{\infty} \mathrm{Col}$ - the low and high limits of the Debye like relaxation region respectively, $\tau_{m}$ - relaxation time calculated by $\tau_{m}=1 / 2 \pi f_{m}$ with $f_{m}$ is relaxation frequency corresponding to the maximum of $\varepsilon^{\prime \prime}(f), \alpha$ - parameter of the relaxation time distribution, $\varepsilon_{\infty}$ lin, $\tau_{\text {lin }}$ and $\alpha_{\text {lin }}$ in equation (3) have the same meaning as $\varepsilon_{\infty} \mathrm{Col}_{0}, \tau_{m}$ and $\alpha$.

The equation (2) was not originated from relaxation theory, it was proved based on the mechanism of domain walls motion in the presence of elastic forces with low viscosity [15]. In the opposite, the linear relaxation region is well described by the equation originated from "universal law" of dielectric relaxation $[15,16]$. In general, with strong viscosity, the elastic force becomes weaker and the motion of domain walls will be no longer reversible. Based on this argument, the assumption of strong-viscosity environment in the synthesized composite seems to be useful to explain the obtained anomalies. Indeed, under the influence of $\mathrm{SiO}_{2}$ on TGS through hydrogen bonds, the viscous striction was increased, inhibited the motion of domain wall, led to rising of the relaxation time and therefore the relaxation frequencies $\left(f_{m}=1 / 2 \pi \tau_{m}\right)$ decreased. As a result, the positions of relaxation peaks $\varepsilon "(f) \mathrm{SiO}_{2}$-TGS composite for all studied samples were shifted toward lower frequencies. At the same time, the enhanced viscosity could lead to the appearance of linear dispersion at higher frequencies as compared to those in bulk of TGS.

Although $\mathrm{SiO}_{2}$ does not contain hydrogen bonds in its structure, the assumption of intermolecular hydrogens between $\mathrm{SiO}_{2}$ nanoparticles and TGS is still reasonable. The reason for that as mentioned above is related to the presence of residual water which can be removed completely from silica only at considerably high temperature $\left(>500{ }^{\circ} \mathrm{C}\right)$ [11]. In the present study, the samples were heated only at 120 ${ }^{\circ} \mathrm{C}$. Moreover, even in the case of good heating treatment at higher $500{ }^{\circ} \mathrm{C}, \mathrm{SiO}_{2}$ can easily absorb moisture from the air under the quite high humidity in Vietnam (about $85 \%$ ). To ensure the stable operation of electronics devices, the samples were stored in real conditions. It worth to note that the presence of water in the composite was confirmed by FTIR patterns by the expansion of $3500-3000 \mathrm{~cm}^{-1}$ [11]. The higher the $\mathrm{SiO}_{2}$ content, the wider the 3500 - 3000 region was detected.

With increasing the $\mathrm{SiO}_{2}$ content, TGS inclusion was well isolated and the $\mathrm{SiO}_{2} / \mathrm{TGS}$ interaction became stronger and could be responsible for the expansion of linear dispersion region at higher $\mathrm{SiO}_{2}$ content as described above. The high isolation of ferroelectrics with high content of dielectric inclusions has been reported by several works as for $\mathrm{SiO}_{2} / \mathrm{TGS}$ or cellulose/Rochelle salt composite [11,17]. In addition, the transformation of linear dispersion into Cole-Cole relaxation with increasing the temperature can be explained by the release of domain wall from the viscous friction.

Another aspect that must be mentioned here is the increase in relaxation frequencies with increasing temperature. Naturally, it was related to thermal activation. During heating process, the viscosity decreased, and therefore the domain walls could be easier to move due to thermal chaos, resulting in the reduction of relaxation time i.e. the relaxation frequencies increased $\left(f_{m}=1 / 2 \pi \tau_{m}\right)$. 
The presence of an additional Cole-Cole arc at room temperature $\left(20^{\circ} \mathrm{C}\right)$ for the mass composition ratio of $\mathrm{SiO}_{2}$ :TGS $=0.2: 1$ (Fig. 2a) obviously corresponds to the volume part of TGS as described above for frequency dependence of the imaginary part of complex permittivity (Fig. 1b). Indeed, for this mass ratio with small $\mathrm{SiO}_{2}$ content, the TGS clusters in large size could be formed. The size effects insignificantly affected and the $\mathrm{SiO}_{2} / \mathrm{TGS}$ interaction became weaker due to the reduction of specific surface areas. As a result, the volume properties took advantages over those on TGS particle surface at room temperature. At higher temperatures, the bulk properties were overlapped by $\mathrm{SiO}_{2} / \mathrm{TGS}$ interactions. This indicates that the TGS clusters or TGS large-size particles have small amount as compared to nanosized TGS particles or TGS that was isolated by $\mathrm{SiO}_{2}$. In some nanocomposites, for example, in cellulose/Rochelle salt composite, Rochelle salt particles were grown in large size with a significant amount and therefore the peaks characteristic for bulk of Rochelle salt disappeared at considerably high content of cellulose in the composite.

The reduction of dielectric constant and dielectric loss with increasing $\mathrm{SiO}_{2}$ content can be explained as follows. The main contribution of dielectric constant obviously came from ferroelectric components, in which the reorientation of dielectric poles of domains plays a leading role. The dielectric inclusion as $\mathrm{SiO}_{2}$ could enhance dielectric/ferroelectric interaction force, but it does not give contribution to dielectric constant. With regard to dielectric loss, the situation is more complicated. Although the increase in $\mathrm{SiO}_{2}$ content led to the accumulation of residual water in the composite, the dielectric loss did not increase. The reason for that might be related to the fact that water molecules in this case play the role of routes, through which the charge carries could be transferred. In addition, there charge carriers were given by TGS components, leading to rising of dielectric loss with increasing TGS content.

The strong blurring of relaxation peaks detected for $\mathrm{SiO}_{2}$ - TGS composite could be counted for the heterogeneity of composite system. Indeed, TGS crystals in the composite were distributed randomly with $\mathrm{SiO}_{2}$ inclusion. The ferroelectricity in TGS particles might be different from each other due to the random orientation of domain walls. As a result, their relaxation processes could be different, leading to the asynchronous response of them under the change of external electric frequency. This behavior is completely consistent with the phase transition features reported in previous work [11], for that the phase transition peaks were blurred strongly. Due to the inhomogeneous distribution of TGS and $\mathrm{SiO}_{2}$ particles, the phase temperatures in TGS particles were not the same, resulting in the long process of phase transition in the whole composite system.

\section{CONCLUSIONS}

Under the influence of silica nanoparitlces with large specific area and high hydrophilicity, the relaxation frequency in triglycine sulfate shifted toward lower frequency range with increasing silica content in the composite. The reason for that is related to the strong interaction between composite components through hydrogen bonds systems, leading to increasing viscosity and slowing down the motion of domain walls. The relaxation mechanism was clarified by Cole-Cole theory based on reversible and irreversible motions of domain walls in TGS crystals. The obtained results suggest an effective method for adjusting electrophysical properties of electronics materials to meet strict requirements in manufacturing industry.

\section{ACKNOWLEDGMENT}

The study was supported by Industrial University of Ho Chi Minh City.

\section{REFERENCES}

[1] A. Hussain, N. Sinha, A. J. Joseph, S. Goel, B. Singh, I. Bdikin and B. Kumar, Mechanical investigations on piezo/ferrolectric maleic acid-doped triglycine sulphate single crystal using nanoindentation technique, Arab. J. Chem., 2018. 
[2] P. R. Deepthi and J. Shanthi, Optical, dielectric \& ferroelectric studies on amino acids doped TGS single crystals, RSC Adv., vol. 6, pp. 33686-33694, 2016.

[3] S. D. Milovidova, O. V. Rogazinskaya, A. S. Sidorkin, H. T. Nguyen, E. V. Grohotova and N. G. Popravko, Dielectric properties of composites based on nanocrystalline cellulose and triglycine sulfate, Ferroelectrics, vol. 469, pp. 116-119, 2014.

[4] H. T. Nguyen, S. D. Milovidova, A. S. Sidorkin and O. V. Rogazinskaya, Dielectric properties of composites based on nanocrystalline cellulose with triglycine sulfate. Phys. Solid State, vol. 57, pp. 503-506, 2015.

[5] H. T. Nguyen, A. S. Sidorkin and S. D. Milovidova, Dispersion of Dielectric Permittivity in a Nanocrystalline Cellulose-Triglycine Sulfate Composite at Low and Ultralow Frequencies, Phys. Solid State, vol. 60, pp. 559$565,2018$.

[6] H. T. Nguyen, A. S. Sidorkin, S. D. Milovidova and O. V. Rogazinskaya, Electrophysical properties of matrix composites nanocrystalline cellulose - triglycine sulfate, Ferroelectrics, vol. 512, pp. 71-76, 2017.

[7] H. T. Nguyen, A. S. Sidorkin, S. D. Milovidova and O. V. Rogazinskaya, Investigation of dielectric relaxation in ferroelectric composite nanocrystalline cellulose - triglycine sulfate, Ferroelectrics, vol. 498, pp. 27-35, 2016.

[8] H. T. Nguyen, A. S. Sidorkin, S. D. Milovidova and O. V. Rogazinskaya, Influence of humidity on dielectric properties of nanocrystalline cellulose - triglycine sulfate composites, Ferroelectrics, vol. 501, pp. 180-186, 2016.

[9] O. M. Golitsyna, S. N. Drozhdin, V. N. Nechaev, A. V. Viskovatykh, V. M. Kashkarov, A. E. Gridnev and V. V. Chernyshev, Dielectric properties of porous aluminum and silicon oxides with inclusions of triglycine sulfate and its modified analogs, Phys. Solid State, vol. 55, pp. 529-535, 2013.

[10] Y.C. Ke and P. Stroeve, Polymer-Layered Silicate and Silica Nanocomposites, Eds. (Elsevier Science, Amsterdam), pp. 69-118, 2005.

[11] B. D. Mai, H. T. Nguyen, T. K. A. Nguyen, D. H. Ta and T. N. Luu, Effects of composition ratio on structure and phase transition of ferroelectric nanocomposites from silicon dioxide nanoparticles and triglycine sulfate, Phase Trans., pp. 1-8, 2019.

[12] S. D. Milovidova, O. V. Rogazinskaya, A. S. Sidorkin, E. V. Vorotnikov, K. T. Nguen and A. P. Lazarev, Dielectric properties of mixed composites prepared from nanodisperse silica and triglycine sulfate, Phys. Solid State, vol. 57, pp. 510-512, 2015.

[13] H.T. Nguyen, Relaxation processes in ferroelectric composites with matrices from nanocrystalline cellulose, Thesis, 115 p., 2016.

[14] A. M. Lotonov, V. K. Novik and N. D. Gavrilova, About dispersion in dielectric permittivity of triglycine sulfate (TGS) ferroelectric in a large temperature interval, Moscow University, vol. 5, pp. 27-32, 2006.

[15] N. M. Galiyarova, Critical slowing down off relaxing domain walls and interfaces in phase transition vicinities, Ferroelectrics, vol. 170, pp. 111-121, 1995.

[16] A. K. Jonscher, The 'universal' dielectric response, Nature, vol. 267, pp. 673-679, 1977.

[17] Hoai Thuong Nguyen and Bich Dung Mai, Study on structure and phase transition of an eco-friendly ferroelectric composite prepared from cellulose nanoparticles mixed with Rochelle salt, Phase Trans., vol. 92, pp. 831-838, 2019. 


\section{SỬ DỊCH CHUYỄN TẦ SỐ TÍCH THOÁT TRONG TRIGLYCINE SUNPHAT DƯới SỰ ẢNH HƯỞNG CỦA HẠT NANO SILICA Ở TẦN SỐ THẤP}

Tóm tắt. Nghiên cứu hiện tại giúp làm rõ sự ảnh hưởng của các hạt nano silica lên tần số tích thoát của một loại vật liệu sắt điện cổ điển - triglycine sunphat ở tần số thấp $\left(10^{2}-10^{7} \mathrm{~Hz}\right)$ từ $20^{\circ} \mathrm{C}$ đến điểm chuyển pha trong các mẫu composite được tổng hợp với các tỉ lệ khối lượng khác nhau. Kết quả nghiên cứu chỉ ra rằng tần số tích thoát giảm khi hàm lượng silica tăng do sự tương tác giữa các hạt nano và triglycine sunphat được tăng cường. Bản chất của tương tác này được giải thích thấu đáo trong nghiên cứu này.

Từ khóa. Ferroelectric nanocomposites, dielectric relaxation, triglycine sulfate, silica nanoparticles, hydrogen bonds. 